\title{
Influence of Solvents on the Oxidation Kinetics of Aldehydic Group Compounds by Diethylammonium Chloro-chromate
}

\author{
DIVYA CHANDORA, PRAMILA BISHNOI, GANPATRAM, OM PRAKASH* \\ and VINITA SHARMA
}

\author{
Chemical Kinetics Laboratory, Department Of Chemistry, J. N. V. University, Jodhpur, \\ 342 005-(Rajasthan), India. \\ *Corresponding author E-mail: drpkvs27@yahoo.com \\ http://dx.doi.org/10.13005/ojc/370609
}

(Received: September 06, 2021; Accepted: December 08, 2021)

\begin{abstract}
The redox studies of some compounds containing aldehydic functional groups by diethylammonium chloro-chromate (DEACC) in dimethylsulfoxide leading a product forming to acid of correspondimg order. Reactions are found to be in unit order with oxidant while a fractional order (less than unity) was found w. r. t. reductants. The redox reactions are influenced with acid, the acid dependence is governed by this equation: $\mathrm{k}_{\text {obs }}=\mathrm{a}+\mathrm{b}\left[\mathrm{H}^{+}\right]$. When isomeric form of aldehyde, that is Me-CDO is oxidised with the same oxidant it was observed a considerable K. I .E. (Deuterium effect; $k_{H} / k_{D}=05.69$ at $298 \mathrm{~K}$ ). The reaction of Acetaldehyde was done in various non aqueous medium, soluble or miscible in DMSO. The effect of solvent is studied fitting our data in the solvent model of Taft's and Swain's applied for this purpose. Rate constants are correlating very well with already reported Taft's values of $\sigma^{*}$; further the reaction constants are negative in nature. Suitable mechanism involving are proposed with transfer of hydride ion.
\end{abstract}

\section{Keywords: Aliphatic aldehydes, Correlation-analysis, DEACC, Oxidation-kinetics, Reaction mechanism.}

\section{INTRODUCTION}

Various dichromates and halochromates have been used time to time as a slow, week and very specific oxidizing agents in the field of imitation organic chemistry ${ }^{1-5}$. Diethylammoniumchlorochromate (DEACC) is also the similar type of compound which is already in use as oxidant for the study of alcohols6. Our group is usually do the oxidation kinetics and is interested to know the mechanism of these redox reactions by complexed chromium compounds and many studies on halochromates and dichromates, including DEACC have already emanated from our this particular laboratory ${ }^{7-10}$ dedicated to the kinetic studies. Not many reactions on the oxidation studies by DEACC are available in therelated literature. as per the experience of our previous work on the subject, we are going to report in the present MS the oxidation kinetics mechanistic studies of some aliphatic aldehydes by DEACC as oxidant and in dimethylsulphoxide (DMSO) as solvent. We have also described the mechanism of this reaction.

This is an Open Access article licensed under a Creative Commons license: Attribution 4.0 International (CC- BY). Published by Oriental Scientific Publishing Company @ 2018 
The following are the salient points to be undertaken by us are as follows: (a) Determination of some kinetic parameter to appraise the rate laws of the reaction, (b) study of effects of the structures on rate and correlation analysis (c) to suggest a very suitable and fit reaction mechanism for this redox reaction.

\section{EXPERIMENTAL}

\section{MATERIAL AND METHODS}

DEACC is synthesised and analysed by the method $^{6}$ already reported in the literature. And the purity of this reported \& synthesised is also checked by an previously established iodometric determination method. $\mathrm{HCHO}$ solutions are also synthesised by warming para-formaldehyde, and fumes are passed into dimethylsulphoxide. Chromotropic acid method ${ }^{11}$ is applied for the determination of formaldehyde in dimethylsulphoxide. All the other aldehydes so used are purchased from the market and are used as received. Para toluene sulfonic acid was in use to provide the acidity. Isomeric acetaldehyde (deuteriated $\mathrm{MeCDO}$ ) is procured as commercial product from Sigma Chemicals. Various other solvents used in the present studies are also purified by usual methods ${ }^{12}$ of their purification available in the literature.

\section{Products analyses}

To find out the product so formed in the end of the reaction is performed in our kinetic reaction conditions the experiments are done with $0.01 \mathrm{~mol}$ acetaldehyde and 0.01 mil dimethyasulfoxide, both are dissolved in $100 \mathrm{~mL} \mathrm{DMSO}$ and this reaction mixture is left for $24 \mathrm{~h}$ to complete the reaction. Than some sodium hydroxide was added to make the solution alkaline in nature. After that reaction mixture is filtered and the filtrate is dried in pressure. Residue so obtained is acidified with some drops of perchloric acid and than an extraction is done with $50 \mathrm{~mL}$ of $5 \% \mathrm{C}_{2} \mathrm{H}_{5}-\mathrm{O}-\mathrm{C}_{2} \mathrm{H}_{5}$. The extract so obtained from ether made moisture free over $\mathrm{MgSO}_{4} \&$ is reacted with a deci $\mathrm{mL}$ of thionylchloride. Solvent so used is evaporated. $7 \mathrm{~mL}$ of Dehydrated methyl alcohol is poured and hydrochloric acid so present is taken out under a flow of moist free air. The remains deposits are going to dissolve in $200 \mathrm{~mL}$ of diethyl ether, and amount of ester is found out by the method of Hall and Schaefer ${ }^{13}$. Various experiments showed 1:1 stoichiometry. lodometric titrations are done to determine the oxidation number of $\mathrm{Cr}$ in reactions mixtures remains at last after completion of reaction. It was found $3.90 \pm 0.15$.

\section{Kinetics-measurement}

To obtain the Pseudo first order reactions conditions the ratio of oxidant over reductant was kept with a higher (x10 or more) of the [reductant] over [oxidant]. Solvent sued in the reactions is DMSO, if changed is mentioned also. Reactions are performed in round bottom flask darkened to avoid any type of photo-chemical reaction. Reactions are performed at a desired temperature which was kept constant also and are followed for 4 half lives. The reactions are monitered by recording the decrease in [reductant] at a lambda max of three hundred and eighty nano-meter $(380 \mathrm{~nm})$. The unit order rateconstant, $k$, is determined by a linearly least square graphs of log [reductant] over time-recorded. To check reproducibility on the rate constant reactions runs are repeated. $k_{2}$, is calculated by dividing the $k$ observed by the concentration of reductant. $k_{2}=$ $\mathrm{k}_{\mathrm{obs}} /$ [aldehyde].

\section{RESULT \& DISCUSSION}

Statistical information through experiments is collected for all the reductants aldehyde and it was observed that results are almost similar, therefore we are representing some of the sample results.

\section{Stoichiometric determination}

Aldehydes are oxidised with DEACC is leading to the carboxylic acid product, which may be shown as the following reaction.

$$
\begin{aligned}
& \mathrm{R}-\mathrm{CHO}+\mathrm{CrO}_{2} \mathrm{ClO}^{-} \mathrm{N}^{+} \mathrm{H}_{2} \mathrm{Et}_{2} \longrightarrow \underset{\mathrm{RCOOH}+}{\mathrm{CrOClO}-\mathrm{N}^{+} \mathrm{H}_{2} \mathrm{Et}_{2}} \\
& \text { (1) }
\end{aligned}
$$

The present oxidant DEACC is showing two electron change in its oxidation state. This similarity has already been seen in previous observation in respect to the similar structural halo- chromates. It has been observed that pyridiniumchlorochromate $(P C C)^{14} \&$ pyridiniumfluorochromate $(P F C)^{15}$ both are acting as two-electron change oxidizing agents and both reduce from chromium (six) to chromium (four). This was recognized by the M.S. (magnetic susceptibility), E.S.R. \& I.R. experiments.

\section{Kinetic dependence}

Most of these reaction so performed 
are of unit orders w. r. t. DEACC. And the unit order rate constant, $\mathrm{k}_{\text {observed }}$ are not dependent to starting concentrations of DEACC. The reactions rate is increasing as we increase the amount of the reductant, but not in the same ratio i. e. not in a linear fashion. It can be observed by the data recorded in table one and by a figure one as a representative graph. Plots of $1 / \mathrm{k}_{\text {obs }}$ versus $1 /$ [reductant] are linearly relation $(r>0.990)$ with a intercept on rate axis. Therefore, fractional order kinetic behaviour is seen w. r. t. aldehyde. This is leading to a postulate following overall mechanism as equation $2 \& 3$ and the kinetic dependence which may be represented as rate-law, equation 4 .

$$
\begin{aligned}
& \text { Aldehyde. + DEACC } \\
& \qquad \begin{array}{l}
\mathrm{K} \\
k_{2}
\end{array} \\
& \text { (Int. Complex.) } \stackrel{\text { Int.Complex })}{\longrightarrow} \text { Products. }
\end{aligned}
$$

Rates $=k_{2} \mathrm{~K}[$ Aldehyde.][DEACC. $] /(1+\mathrm{K}[$ Aldehyde. $])$

Table 1: Constant of rates for the oxidation of Me-CHO by DEACC at $288 \mathrm{~K}$

\begin{tabular}{ccc}
\hline $\begin{array}{c}10^{3} \text { [DEACC] } \\
\text { Mol. Dm/10 }\end{array}$ & $\begin{array}{c}\text { [Aldehyde] } \\
\text { Mol. Dm/10 }\end{array}$ & $\begin{array}{c}10^{4} k_{\text {obs }} \\
\text { Per Second }\end{array}$ \\
\hline 1.00 & .10 & 9.39 \\
1.00 & .20 & 13.7 \\
1.00 & .40 & 17.7 \\
1.00 & .60 & 19.7 \\
1.00 & .80 & 20.8 \\
1.00 & 1.0 & 21.6 \\
1.00 & 1.5 & 22.7 \\
1.00 & 3.0 & 23.9 \\
2.00 & .20 & 12.6 \\
4.00 & .20 & 14 \\
6.00 & .20 & 13.5 \\
8.00 & .20 & 13 \\
1.00 & .40 & $18.0^{*}$ \\
\hline
\end{tabular}

${ }^{\mathrm{a}}$ contained $0.001 \mathrm{M}$ acrylonitrile

We have studied the reliance of our reaction-rate on the aldehyde concentrations at various changed temperature. Data for formation constants and decomposition constants are calculated from the plots shown in graph two. Data for the thermodynamic parameter of the complex so formed during the reaction and the activation parameter of the decomposition of these complex are also evaluated with the result of $K$ and $k_{2}$ respectively at various temperature which are shown in tables two and three.
Table 2: Rate constants \& the activation parameters

\begin{tabular}{|c|c|c|c|c|c|c|c|}
\hline \multirow[b]{2}{*}{ Aldehyde } & \multicolumn{4}{|c|}{$104 k_{2} /\left(\mathrm{dm}^{3} / \mathrm{mol} / \mathrm{s}\right)$} & \multirow{2}{*}{$\begin{array}{c}\Delta . H^{*} \\
(\mathrm{~kJ} / \mathrm{mol})\end{array}$} & \multirow{2}{*}{$\begin{array}{c}-\Delta \cdot \mathrm{S}^{*} \\
\left(\mathrm{~J} \mathrm{~mol}^{-1} \mathrm{~K}^{-1}\right)\end{array}$} & \multirow{2}{*}{$\begin{array}{c}\Delta . \mathrm{G}^{*} \\
(\mathrm{~kJ} / \mathrm{mol})\end{array}$} \\
\hline & 288 & 298 & 308 & 318.K & & & \\
\hline $\mathrm{H}$ & 2.43 & 5.58 & 12.6 & 27 & $58.7 \pm 0.4$ & $111 \pm 1$ & $91.5 \pm 0.3$ \\
\hline $\mathrm{Me}$ & 25.2 & 52.2 & 108 & 207 & $57.1 \pm 0.3$ & $118 \pm 1$ & $86.0 \pm 0.2$ \\
\hline Et & 40.5 & 82.8 & 162 & 306 & $48.8 \pm 0.1$ & $122 \pm 1$ & $84.9 \pm 0.1$ \\
\hline $\mathrm{Pr}$ & 43.2 & 88.2 & 180 & 333 & $49.6 \pm 0.4$ & $118 \pm 1$ & $84.7 \pm 0.3$ \\
\hline Pri & 62.1 & 126 & 243 & 450 & $47.7 \pm 0.1$ & $122 \pm 1$ & $83.9 \pm 0.1$ \\
\hline $\mathrm{ClCH}_{2}$ & 0.17 & 0.45 & 1.17 & 2.7 & $67.9 \pm 0.3$ & $101 \pm 1$ & $97.8 \pm 0.3$ \\
\hline MeCDO & 4.23 & 9.18 & 19.8 & 39.6 & $54.4 \pm 0.3$ & $121 \pm 1$ & $90.3 \pm 0.2$ \\
\hline $\mathrm{k}_{\mathrm{H}} / \mathrm{k}_{\mathrm{D}}$ & 5.96 & 5.69 & 5.45 & 5.23 & & & \\
\hline
\end{tabular}
for oxidation of some aliphatic aldehydes-DEACC complex

Table 3: Complex Formation constants and the thermodynamic parameter of aliphatic aldehydes -DEACC complex

\begin{tabular}{llllllll}
\hline & \multicolumn{3}{c}{$K\left(\mathrm{dm}^{3} \mathrm{~mol}^{-1}\right)$} & & $-\Delta . \mathrm{H}^{*}$ & $-\Delta . \mathrm{S}^{*}$ & $-\Delta . \mathrm{G}^{*}$ \\
Aldehyde & 288 & 298 & 308 & $318 K$ & $\left(\mathrm{k} . \mathrm{J} \mathrm{mol}^{-1}\right)$ & $\left(\mathrm{J} \mathrm{mol}^{-1} \mathrm{~K}^{-1}\right)$ & $\left(\mathrm{k} . \mathrm{J} \mathrm{mol}^{-1}\right)$ \\
\hline $\mathrm{H}$ & 6.03 & 5.45 & 4.77 & 4.17 & $12.0 \pm 0.4$ & $18 \pm 1$ & $6.66 \pm 0.3$ \\
$\mathrm{Me}$ & 5.94 & 5.3 & 4.68 & 4.08 & $11.9 \pm 0.3$ & $19 \pm 1$ & $6.61 \pm 0.2$ \\
$\mathrm{Et}$ & 5.85 & 5.25 & 4.59 & 3.99 & $12.2 \pm 0.4$ & $20 \pm 1$ & $6.57 \pm 0.3$ \\
$\mathrm{Pr}$ & 6.12 & 5.52 & 4.86 & 4.2 & $12.0 \pm 0.5$ & $18 \pm 2$ & $6.91 \pm 0.4$ \\
$\mathrm{Pri}$ & 5.76 & 5.15 & 4.52 & 3.87 & $12.5 \pm 0.5$ & $21 \pm 2$ & $6.52 \pm 0.4$ \\
$\mathrm{ClCH}_{2}$ & 5.67 & 5.05 & 4.41 & 3.8 & $12.6 \pm 0.4$ & $21 \pm 1$ & $6.48 \pm 0.3$ \\
$\mathrm{MeCDO}$ & 5.58 & 4.97 & 4.32 & 3.62 & $13.4 \pm 0.6$ & $24 \pm 2$ & $6.42 \pm 0.5$ \\
\hline
\end{tabular}

\section{Induced polymerization test}

Aliphatic aldehyde under inert ambience created by the $\mathrm{N}_{2}$ gas, are nor subjected to the polymerisation by acrylonitrile to reassure the free radical test an added acrylonitrile also did not affect rate of the reaction. This is an indication that reaction is not run by free radical mechanism and one electron oxidation process is not observed in this present reaction. Absence of free radical is further reconfirmed by carrying out the reaction with $\mathrm{BHT} /$ butylated-hydroxy toluene. The recovery of unaffected $\mathrm{BHT}$ also confirms that no free radical mechanism is there.

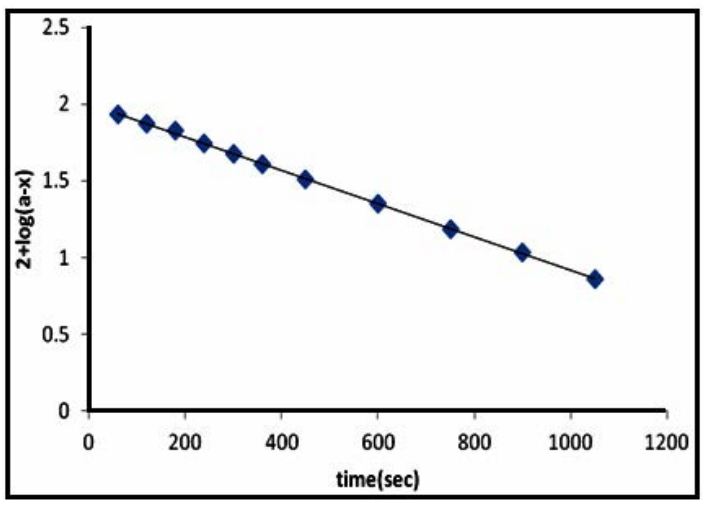

Fig. 1. Typical kinetic run of Oxidation of Me-CHO by DEACC 


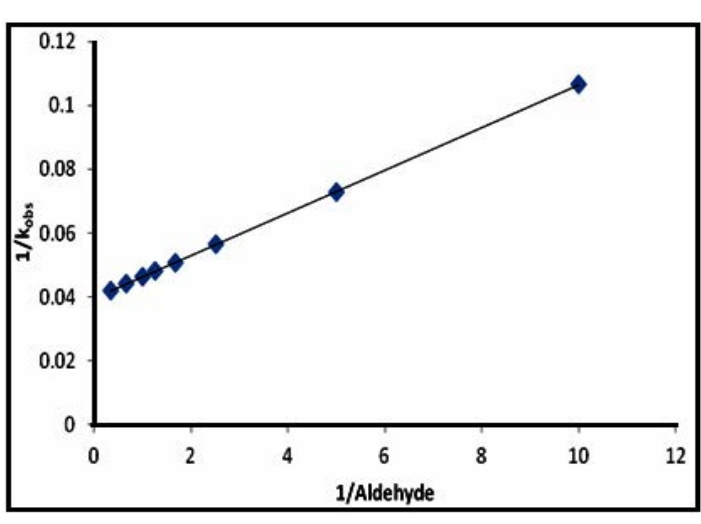

Fig. 2. $1 / \mathrm{k}_{\text {obs }}$ Vs $1 /$ Ald graph of Me-CHO, oxidised by DEACC

\section{Deuterium isotope effect on oxidation}

To establish the significance of break-up in aldehyde Carbon-Hydrogen bond in the step which determines the rate, oxidation of Me-CDO is taken under studies. A considerable preliminary Deuterium effect is seen in oxidizing process of Me-CDO. Results in Table 3 are given above.

\section{Influence of hydrogen ions in reaction rate}

It may be noted here that rate of the reaction is catalyzed and raised with the rise in the presence concentrations of hydrogen ion., Table 4. Influence of hydrogen ion concentrations are having the expression as k-observe $=\mathrm{a} .+\mathrm{b} .\left[\mathrm{H}^{+}\right]$. The data for $a$. and $b$, for $\mathrm{Me}-\mathrm{CHO}$, is found to be $9.16 \pm$ $0.29 \times 10^{-4} \mathrm{~s}^{-1}$ and $15.5 \pm 0.49 \times 10^{-4} \mathrm{~mol}^{-1} \mathrm{dm}^{3} \mathrm{~s}^{-1}$ $(r=0.9980)$.

Rate $=k_{2}$ [DEACC] [Aldehydes] $+k_{3}$ [DEACC]
[Aldehydes] [TsOH]

Table 4: Influence of the hydrogen ion on the reactions rates

\begin{tabular}{lcccccc}
\hline$[D E A C C$ & $=0.001$ & \multicolumn{3}{c}{ mol. $\mathrm{dm}^{-3} ;$} & \multicolumn{3}{c}{ [Aldehydel] $=1.0$} & $\mathrm{~mol}^{-3} \mathrm{dm}^{-3} ;$ & \multicolumn{2}{c}{ Temperature $=288 \mathrm{~K}$} \\
\hline$\left[\mathrm{H}^{+}\right] / \mathrm{mol} \mathrm{dm}^{-3}$ & 0.1 & 0.2 & 0.4 & 0.6 & 0.8 & 1 \\
$10^{4} \mathrm{k}_{\text {obs }} / \mathrm{s}^{-1}$ & 10.8 & 12.6 & 15.3 & 18 & 21.6 & 25.2 \\
\hline
\end{tabular}

\section{Effects of various solvent on reaction rate}

Oxidation studies of $\mathrm{Me}-\mathrm{CHO}$ are done in nine-teen various non-aqueous compounds as solvating agents. Because of nature of solvation of
DEACC the selection of solvents found to be limited. The kinetics in all the solvents are found to be similar the data for rate \& formation constants so collected are given in table given below.

Table 5: Influence of Solvent over oxidation of MeCHO-DEACC complex at $298 \mathrm{~K}$

\begin{tabular}{|c|c|c|c|c|c|}
\hline Medium & $\mathrm{K}\left(\mathrm{dm} / 10^{3} / \mathrm{mol}\right)$ & $10^{4} \mathrm{k}_{\mathrm{obs}} / \mathrm{S}$ & Medium & $\mathrm{K}\left(\mathrm{dm} / 10^{3} / \mathrm{mol}\right)$ & $10^{4} \mathrm{k}_{\mathrm{obs}} / \mathrm{S}$ \\
\hline $\mathrm{CHCl}_{3}$ & 5.55 & 16.6 & $\mathrm{C}_{6} \mathrm{H}_{5} \mathrm{CH}_{3}$ & 5.57 & 4.27 \\
\hline 1,2-Di-chloroethane & 5.80 & 18.6 & $\mathrm{CH}_{3}-\mathrm{CO}-\mathrm{C}_{6} \mathrm{H}_{5}$ & 5.29 & 22.4 \\
\hline Di-chloromethane & 6.00 & 15.1 & THF & 4.92 & 7.41 \\
\hline DMSO & 5.30 & 52.2 & t-Butyl alcohol & 5.60 & 7.08 \\
\hline $\mathrm{Ch}_{3}-\mathrm{CO}-\mathrm{CH}_{3}$ & 6.03 & 14.5 & 1,4-Di-oxane & 5.50 & 7.94 \\
\hline Dimethylformamide & 5.45 & 29.5 & 1,2-Di-methoxyethane & 5.75 & 4.57 \\
\hline Butanone & 5.81 & 10.5 & Carbon disulfide & 6.00 & 2.14 \\
\hline $\mathrm{C}_{6} \mathrm{H}_{5} \mathrm{NO}_{2}$ & 5.72 & 20.4 & $\mathrm{CH}_{3}-\mathrm{COOH}$ & 5.71 & 3.47 \\
\hline $\mathrm{C}_{6} \mathrm{H}_{6}$ & 5.46 & 5.25 & $\mathrm{C}_{6} \mathrm{H}_{5} \mathrm{COCH}_{3}$ & 5.81 & 6.46 \\
\hline Cyclo-hexane & 6.12 & 0.51 & & & \\
\hline
\end{tabular}

Satisfactory correlation $(r=0.9915)$ between $\Delta \mathrm{H}$ and $\Delta \mathrm{S}$ of oxidation of all the aldehydes under study indicated the application of effect of compensation in the present reaction ${ }^{16}$. The degree of isokinetic temperature so evaluated ${ }^{17,18}$ by this plot is found to be in the tune of $965 \pm 63$ $K$. The correlation between all the aldehydes is analysed and observed and found authentic by the Exners' principle ${ }^{19}$. Correlation in the calculated data of enthalpy \& entropy is mostly not match with investigational errors related with. Temperature, known to be isokinetic was intended from Exner's principle of $\log k_{2}$ at lower versus $\log k_{2}$ at higher temperatures respectively $(r=0.9998)$ is $998 \pm 37$ $K$ (Fig. 3). Linearity in the isokinetic temperature correlations indicated that compounds under study may be oxidizing as per the similar process.

\section{Influence of Solvents}

Influence of solvent was studied and rate constants are correlated with LSER (Equation 6) of Kamlets and his group ${ }^{26}$. Only 18 solvents are taken into consideration of correlation because of non-availability of complete set of parameter of carbon disulphide. 
Log $-k_{2}=A_{0}+p \times \pi^{\star}+b \times \beta+a \times \alpha$

(6)

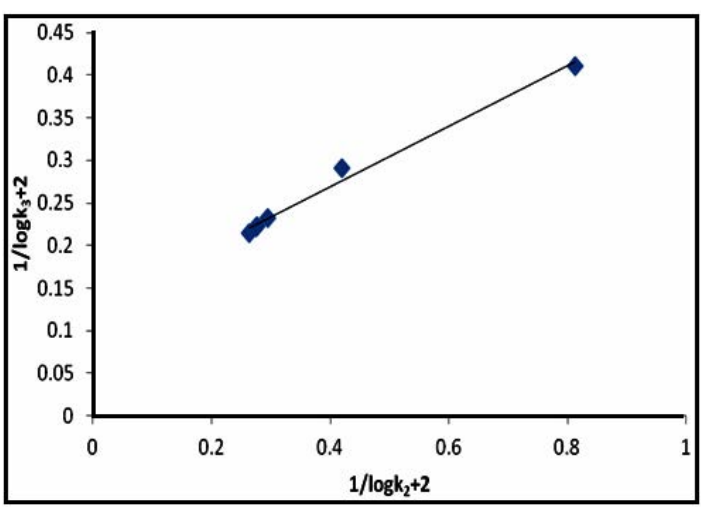

Fig. 3. Plot by Exner principle of Isokinetic Relationship

In present model of reaction

$\pi^{*}$ denotes the solvent-polarity. $\beta$ represented basicity of hydrogen bond acceptor capacity., a denotes acidity of hydrogen bond donor capacity. And intercept is $A_{0}$ in the equation. It can be stated at this point that out of the eighteen solvent, twelve are having the data naught for $\alpha$. Data of present correlation-analysis calculated with the help of equation 6 , involving $\pi^{\star} \& \beta$, and individually with $\pi^{\star} \& \beta$ are represented in equations 7 to 10 .

$\log -k_{2 .}=-4.18+1.66( \pm 0.20) \pi^{\star}+0.20( \pm 0.17) \beta+$ $0.04( \pm 0.16) \alpha$

$\mathrm{R}_{2}=0.8588 ; \mathrm{SD}=0.19 ; \mathrm{n}=18 ; \psi .=0.41$

$\log -k_{2}=-4.19+1.67( \pm 0.19) \pi^{\star}+0.19( \pm 0.15) \beta$

$\mathrm{R}_{2}=0.8582 ; \mathrm{SD}=0.18 ; \mathrm{n}=18 ; \psi .=0.40$

Log $-k_{2}=-4.15+1.72( \pm 0.18) \pi^{*}$

$\mathrm{r}^{2}=0.8437 ; \mathrm{SD}=0.18 ; \mathrm{n}=18 ; \psi .=0.41$

$\log -k_{2}=-3.22+0.49( \pm 0.36) \beta$

$r^{2}=0.1013 ; S D=0.44 ; n=18 ; \psi .=0.97$

Where $n$ is representing numeral of data under calculation and $\psi$ known as Exner's mathematical parameter ${ }^{21}$.

Triparametric equation $\mathrm{Kamlet}^{20}$ is explaining almost $86 \%$ of influence on rates of reaction process. But as per the Exner's criterion ${ }^{21}$ correlation is not satisfactory as shown in equation 7. Where main role is contributed by solvent polarity. The single contribution of solvent polarity is found to be $84 \%$ of the data. $\beta$ and $\alpha$ are relatively playing a very relatively negligible role.

The same set of experimental data of the effect of solvents are also analysed and fitted into next model of Swain's equation ${ }^{22}$ which is based on cation \& anion solvation perception of the solvent as shown below.

$\log -k_{2}=a \times A+b \times B+. C$.

In this equation $A$ is representing anion solvating power, $\mathrm{B}$ is denoting cation solvating power. And $C$ here is represented by cut off expression. Expression $(A+B)$ is representing polarities of solvents. Rates in various solvent are calculated in sa per the eq. 8 and singly with $A$ \& $B$ and with $(A+B)$ also.

$$
\begin{aligned}
& \text { Log }-k_{2}=0.79( \pm 0.03) \mathrm{A}+1.72( \pm 0.02) \mathrm{B}-4.40 \\
& \mathrm{R}^{2}=0.9967 ; \mathrm{SD}=0.03 ; n=19 \psi=0.06 \\
& \text { Log }-k_{2}=0.55( \pm 0.57) \mathrm{A}-3.22 \\
& \mathrm{r}^{2}=0.0515 ; \mathrm{SD}=0.46 ; n=19 \psi=1.00 \\
& \log -k_{2}=1.66( \pm 0.14) \mathrm{B}-4.15 \\
& \mathrm{r}^{2}=0.8898 ; \mathrm{SD}=0.16 ; n=19 \psi .=0.34 \\
& \log -k_{2}=1.42 \pm 0.12(\mathrm{~A}+\mathrm{B})-4.37 \\
& \mathrm{r}^{2}=0.8895 ; \mathrm{SD}=0.16 ; n=19 \psi .=0.34
\end{aligned}
$$

Oxidation rate of acetaldehyde in various solvents prove to be very good correlation in the equation (12) of Swain's model. Here salvation capacity of positive charged ions having dominating function. Particularly, cation solvating power alone is responsible for $89 \%$ of the data obtained. Further, it is observed that correlation is highly poor with negative charged ion solvating capacity power of the solvent. Solvent polarity, $(A+B)$ is also justifying our. $88 \%$ results. It is apparent that when the $A+B$ is explaining $88 \%$ results, we correlated the rates with relative permittivity of Solvent. Agraph of rate constant with contrary of the relativepermittivity is not found to be linear e.g. $r=0.5299$; $\mathrm{SD}=0.33 ; \psi=0.71$.

\section{Reactivity-Correlation analysis of aldehydes with DEACC}

Oxidation rates of all the six aldehydes under study showed a very good correlation with $\pi^{\star}$ substituent constants of $\mathrm{Taft}^{23}$, with reaction constants being negative. Data given in Table 6. -ve polar reaction constants are the indication that low electron density carbon centre is present in slow step of the reaction mechanism. 
Table 6: Dependence of temperatures on the polar reaction constants

\begin{tabular}{lcccc}
\hline Temperature & $-\rho^{*}$ & $\mathrm{r}^{2}$ & $\mathrm{SD}$ & $\psi$. \\
\hline $288 \mathrm{~K}$ & $2.07 \pm 0.01$ & 0.9999 & 0.002 & 0.02 \\
$298 \mathrm{~K}$ & $1.97 \pm 0.01$ & 0.9998 & 0.005 & 0.01 \\
$308 \mathrm{~K}$ & $1.87 \pm 0.02$ & 0.9989 & 0.019 & 0.01 \\
$318 \mathrm{~K}$ & $1.79 \pm 0.01$ & 0.9999 & 0.014 & 0.02 \\
\hline
\end{tabular}

\section{Mechanisistic path way}

Dependence on hydrogen ions so observed is suggesting that present reaction is following two mechanistic pathway, first is independent on acid effect and the other is dependent on acid. By catalytic effect of acid, DEACC is protonated and acts as a strong oxidizing agent and and electrophile also, as shown in equation 16. Both unprotonated and protonated DEACC are acting as reactive oxidizing species. Out of which protonated species proved to be more reactive.

$$
\mathrm{CrO}_{2} \mathrm{ClO}^{-} \mathrm{N}^{+} \mathrm{H}_{2} \mathrm{Et}_{2}+\mathrm{H}^{+} \leftrightharpoons\left[\mathrm{HOCrOClO}^{-} \mathrm{N}^{+} \mathrm{H}_{2} \mathrm{Et}_{2}\right]
$$

Protonated chromium (VI) formation are reported in previous studies also in the reaction of similarly structural compounds like morpholinium chlorochromate ${ }^{24}$ and tetraethylammonium chlorochromate ${ }^{25}$.

It has already been established that most of the aliphatic aldehydes primarily remain in the hydrate form ${ }^{26}$ in presence of water in the reaction medium. In so many oxidation reactions in water containing media, it is already reported to have that hydrate form of aldehyde is taking part in the reaction. But our reactions are done in non-aqueous solvents, therefore, her only free carbonyl form of aldehyde can be taken into consideration as reactive species.

We have no evidence which can prove the formation of an intermediate in this reaction but we also can not reject itys formation in very small amount. Considerable amount of primary-KIE $\left(\mathrm{k}_{\mathrm{H}} / \mathrm{k}_{\mathrm{D}}=5.96\right.$ at $288 \mathrm{~K}$ ) is confirming that Carbon Hhdrogen bond in aldehyde is cleaving in week speed step that is $r$. d. s.

Big -ve values of $-\rho^{*}$ and considerable kinetic isotope effect is indicating that the transition state is approaching towards a carbon having positive charge in its nature. Therefore, a hydride- ion transfer from reductant (aldehyde) to DEACC, is proposed. This particular reaction mechanism may also be supported by noteworthy involvement of positive charged ion solvating capacity of the solvent media. On the observations and results obtained, reaction mechanism given below as schemes 1 and 2 .
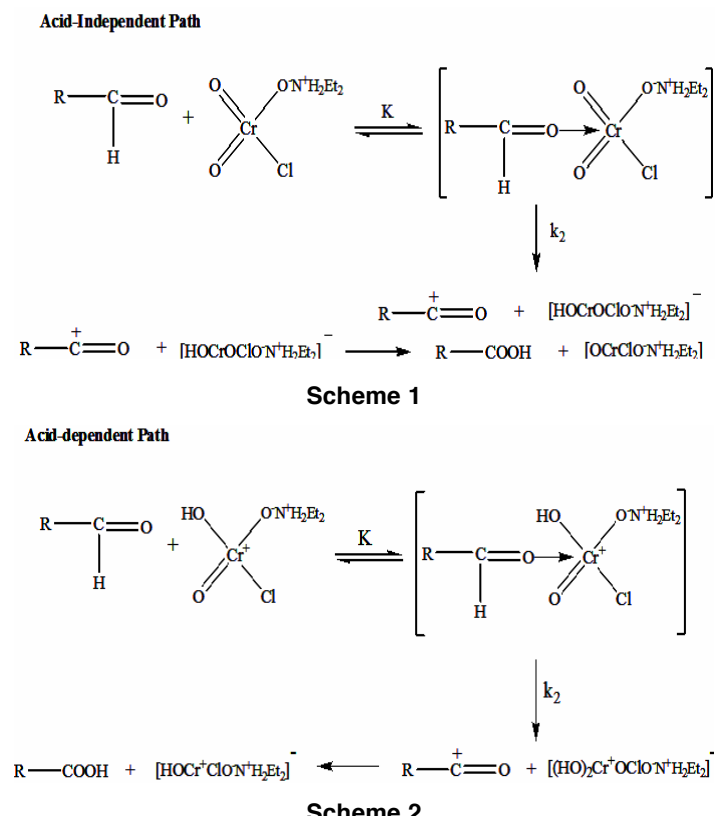

If one compares the oxidizing process of these reductants with $\mathrm{PFC}^{27}, \mathrm{PBC}^{28}, \mathrm{QFC}^{29}$ and DEACC. Oxidation by QFC, PFC and DEACC are presenting almost similar way of kinetic and mechanistic depiction.

A Michaelis menten type kinetics, w. r. t. aldehydes are observed, whereas by the $\mathrm{PBC}^{28}$ reactions are found to first order w. r. t. reductants. Therefore, it looks that the data of complex formation constant for intermediates are very small in case of PBC. This particular behaviour of the reaction goes in favour of second order kinetics. All other parameters such as kinetic isotope effects, effect of solvents and the effect of acidity are of comparable in these systems. Workers have proposed almost equal reaction path ways to these reactions.

\section{ACKNOWLEDGEMENT}

OP is thankful of University Grants Commission, New-Delhi, India, for monitory support as UGC-Basic Scientific Research Start-up Grant, authors are also thankful to HOD Chemistry, Jai Narain Vvyas University, Jodhpur for lab facilities. 


\section{REFERENCES}

1. Corey, E. J.; Suggs, W. J. Tetrahedron Lett., 1975, 2647.

2. Guziec, F.S.; Luzio, F.A. Synthesis., 1980, 691

3. Bhattacharjee, M.N.; Choudhuri, M.K.; Dasgupta, H.S.; Roy, N.; Khathing, D.T. Synthesis., 1982, 588.

4. Balasubramanian, K.; Prathiba, V. Indian J. Chem., 1986, 25B, 326.

5. Pandurangan, A.; Murugesan, V.; Palamichamy, P. J. Indian Chem. Soc., 1995, 72, 479.

6. Sinhg, V.; Mahajan, S.; Jasrotia, V.S.; Sharma, M.; Sheikh, H. N.; Kalsotra, B. L. J. Indian Chem. Soc., 2009, 84, 528.

7. Vyas, N.; Goswami, G.; Choudhary, A.; Prasadrao, P. T. R. S. K.; Sharma, V. Int. J. Chem., 2015, 4(3), 215.

8. Sharma, J.; Singadiya, A.; Prakash, O.; Sharma, V. J. Emer. Tech. Innov. Res., 2021, 8(4), 167.

9. Vyas, N.; Rao, A.; Purohit, P.; Sharma, V. J. Inter. Cycle Res., 2021, 13(3), 1377.

10. Kamla. Naga, P.; Purohit, P.; Sharma, V. Rasayan J. Chem., 2021, Accepted; MS No. RJC-6587-2021.

11. Mitchell J. Jr., Organic analysis, Interscience, New York, 1954, II, 273.

12. Perrin D. D., Armarego W. L., Perrin D. R., Purification of organic compounds, Oxford, Pergamon Press., 1966.

13. Hall, R.T.; Schaefer, W.E., Organic Analysis, Interscience New York., 1954, II, 55.
14. Brown, H.C.; Rao, G.C.; Kulkarni, S.U. J. Org. Chem., 1979, 44, 2809.

15. Bhattacharjee, M.N.; Choudhuri, M. K.; Purakayastha, S. Tetrahedron., 1987, 43, 5389.

16. Liu, L.; Guo, W. E. Chem. Review., 2001, 101,673.

17. Leffler, J. E. J. Org. Chem., 1955, 20, 1202; J. Phys. Chem., 1964, 29, 2199.

18. Patterson, R.C. J. Org. Chem., 1964, 29, 3133.

19. Exner O., Collect. Chem. Czech. Commun., 1964, 29, 1094.

20. Kamlet, M. J.; Abboud, J L M.; Abraham, M. H.; Taft, R. W.; J. Org. Chem., 1983, 48, 2877.

21. Exner, O. Collect. Chem. Czech. Commun., 1966, 31, 3222.

22. Swain, C. G.; Swain, M. S.; Pqwel, A. L.; Alunni, S. J. Am. Chem. Soc., 1983, 105, 502.

23. Taft R. W., Steric effects in organic chemistry, Ed. M.S. Newman, Wiley, New York., 1956.

24. Choudhary, A.; Malani, N.; Agarwal, A.; Sharma, M.; Sharma, V. J. Indian Chem. Soc., 2009, 86, 927.

25. Gehlot, M.; Prasadrao, PTSRK.; Sharma, V. Asian J. Chem., 2011, 23(3), 1173.

26. Bell R. P. Adv. Phys. Org. Chem., 1966, 4, 1.

27. Agarwal, A.; Choudhary, K.; Banerji, K.K. J. Chem. Res., 1990, 86.

28. Khanchandani, R.; Sharma, P. K.; Banerji, K. K. Indian J. Chem., 1996, 35A, 576.

29. Khurana, M.; Sharma, P. K.; Banerji, K. K.; React. Kinet. Catal. Lett., 1999, 67, 341. 